\title{
Role of AP2/EREBP Transcription Factor Family in Environmental Stress Tolerance
}

\author{
Pande A로 Saxena SC ${ }^{3}$, Thapliyal M11, Guru SK ${ }^{2}$, Kumar A² and Arora \\ $\mathrm{S}^{1 *}$ \\ ${ }^{1}$ Department of Molecular Biology and Genetic Engineering, G. B. Pant University of \\ Agriculture and Technology, India \\ 2Department of Plant Physiology, G. B. Pant University of Agriculture and \\ Technology, India \\ ${ }^{3}$ Delhi Technical University, India
}

*Corresponding author: Sandeep Arora, Department of Molecular Biology and Genetic Engineering, College of Basic Sciences and Humanities, G. B. Pant University of Agriculture and Technology, Pantnagar, Uttarakhand, India, Tel: 7055470555; Email: plantstress@gmail.com

\begin{abstract}
Plants being sessile in nature have to face several adverse environmental conditions throughout their life cycle. The adverse environmental conditions include both abiotic and biotic stresses. Plant responses to these stresses are complex and involve numerous physiological, molecular, and cellular adaptations. Their perception and response is highly spontaneous and involves the production of important metabolic proteins such as those involved in the synthesis of osmo-protectants, chaperons, antioxidants and regulatory proteins operating in the signal transduction pathways. It is now a common knowledge that stress response is a multi-gene program, with several metabolic pathways being involved in cumulative responses. The stress response is a highly dynamic process and depends upon the magnitude as well as the multitude of stresses. As a result, multiple stress signal perception and transduction pathways are involved, which may also cross talk at various levels. Therefore, plants possess an intricate defence mechanism, involving multiple gene regulatory pathways. Transcription factors play a critical role in such defence responses by coordinating the expression of genes that are involved in the defence pathway to overcome the stress load in plants. AP2 (APETALA2)/EREBP (Ethylene-Responsive Element-Binding Protein) family of transcription factor is one of the largest in plants and has important regulatory functions in environmental stress tolerance. Furthermore, AP2/EREBP family also regulates diverse processes of plant development and metabolism such as vegetative and reproductive development, cell proliferation, secondary metabolism and responses to different plant hormones. This review focuses on the recent advances in exploring the role of AP2/EREBP transcription factor family proteins in environmental stress responses in plants.
\end{abstract}

Keywords: APETALA-2; Environmental Stresses; Transcription Factors; Transgenic Plants 


\section{Cell \& Cellular Life Sciences Journal}

Abbreviations: EREBP: Ethylene-Responsive Element-Binding Protein; TFs: Transcription Factors; RWC: Relative Water Content; ABA: Abscisic Acid; DREB: Dehydration-Responsive Element Binding.

\section{Introduction}

In nature plants are exposed to a variety of environmental stresses that adversely affect their growth and productivity. Plants deal with such stressful environmental conditions by enduring an array of physiological and morphological alterations, needed for their survival. A combination of intrinsic homeostatic changes ascertains the capacity of a plant to sustain itself under unfavorable environmental conditions. Plant response to imposed stresses may involve passive tolerance or active balancing homeostatic mechanisms. Some of the common plant responses to environmental stresses range from leaf area reduction, root growth stimulation, alterations in relative water content (RWC), to stimulation and accumulation of antioxidants like ascorbate \& phenolic compounds, compatible osmolytes like proline etc. Molecular responses to abiotic stress, on the other hand, include stress signal perception, signal transduction, alterations in gene expression and ultimately metabolic changes in the plant, thus providing stress tolerance [1]. To this effect, transgenic technology has been one of the main stay for development of new varieties that have the ability to sustain growth and productivity under stressful environment. The last few decades have seen a drastic change in the strategies for development of transgenic plants. In the initial phases, scientists focused on the use of individual genes and proteins for development of stress tolerance attribute [2]. However, with an improved understanding of the molecular biology of stress tolerance in plants, the focus has shifted to the use of plant growth regulators and transcription factors for developing stress tolerant transgenic crops.

\section{Role of Transcription Factors}

Transcription factors (TFs) play an important role in regulating gene expression in plants and are rightly referred to as the master regulators of the cell. These factors play an active role in initiating the transcription process as well as regulating the developmental and environmental response signals during the whole life cycle of an organism. They are capable of triggering a unique array of metabolic reactions, by modifying the transcript profile of a plant cell. For crop improvement, elucidating the molecular mechanisms underlying the activation/ repression of specific genes through the binding of transcription factors is of utmost importance. These metabolic adjustments help plants to respond to the challenging environmental conditions, since plants are sessile and cannot escape from environmental stresses. Several transcription factors are important candidates for adapting plants to external stress, since they regulate the expression of a number of stress responsive genes, the regulons, by binding to their specific cis-element promoters. The important role played by various transcription factors, in the life cycle of a plant is evident from the fact that a collection of more than 2000 known and predicted rice (Oryza sativa) transcription factors, distributed amongst 63 families is available in the database of rice transcriptional factors [3].

Transcription factors are basically sequence-specific DNA-binding proteins that are capable of activating and/or repressing the transcription process. One of the largest families of transcription factor is the AP2/EREBP (APETALA 2/ethylene responsive element-binding protein). Many transcription factors belonging to this family play important regulatory and developmental role in plant kingdom. Regulons controlled by AP2/ERF (apetala2/ethylene Responsive factor), ABF/AREB (abscisic acid binding factor/abscisic acid responsive element binding) and NAC (NAM, ATAF and CUC domain containing) class of transcription factors are specifically involved in abiotic stress responses [4]. This family of transcription factors is known to be involved in biotic stress response via the Jasmonic acid pathway and in abiotic stress response via the ABA biosynthetic pathway [5]. However, whole genome expression analysis of stress inducible genes in Arabidopsis indicates the existence of interconnected regulatory networks between stress signal perception and expression of stress responsive genes [6]. Over-expression of the gene that regulates transcription of a number of downstream responsive genes seems to be a promising strategy for the development of new varieties with environmental stress tolerance attributes.

\section{Involvement of Multitude of Transcription Factors in Environmental Stress Responses}

Recent advances in the science of omics have brought notable progress in elucidating the role of various transcription factors in plant stress tolerance response. By utilizing modern genetics and functional genomics approaches such as transcriptomics, proteomics and metabolomics, various stress responsive genes and proteins have been identified and characterized in crops [7]. Transcription factors are reported to regulate the 


\section{Cell \& Cellular Life Sciences Journal}

activity of specific proteins/ enzymes that participate in metabolic processes such as those involved in detoxification, osmolyte biosynthesis, proteolysis, ion channel functioning, ion sequestering, heat and cold shock proteins, late embryogenesis abundant protein etc [8]. Major factors having regulatory role, primarily comprises of AREB, AP2/ERF, NAC, bZIP, MYC, and MYB transcription factors. Another class of important molecules involved in regulatory \& signalling role is the kinases e.g. mitogen activated protein kinases, calciumdependent protein kinases, receptor protein kinases, ribosomal protein kinases, and transcription regulation protein kinases. These signalling kinases coordinate stress signal perception, transduction and response [8]. Among the various regulatory proteins, stress-responsive TFs have evoked widespread interest for their role in plant stress tolerance. Several researchers have used transcription factor genes to develop transgenic plants that have met with mixed success.

During the last decade a number of transcription factor families, implicated in environmental stress tolerance have been studied. During exposure to stress these transcription factors act as molecular switches. In plants, a significant per cent of total genome (upto10\%) potentially codes for transcription factors. In Arabidopsis nearly $6 \%$ of the proteome is dedicated to transcription factors. These transcription factors mainly regulate $\mathrm{ABA}$ dependent or ABA independent stress responsive pathways. Several families of these transcription factors e.g. AREB, DREB, MYB, WRKY, NAC, and bZIP have been implicated in environmental stress responses; and these transcription factors have been categorized based on the distinct structure of their DNA- binding motifs [8]. Several of these transcription factors overlap functionally, during a plant's response to environmental stresses. This fact is corroborated by the observations that several abiotic stresses have distinct primary effects, but common secondary effects. Further a widespread overlap between other signal transducing molecules and the transcription factors could be both synergistic and antagonistic, resulting in positive or negative feedback loops.

Interactions amongst different transcription factors, with respect to their role in stress tolerance, have been studied extensively over the years and are documented by Nakashima, et al. [9]. The transcription factors control transcription of their downstream genes in conjunction with other proteins by binding to the conserved sequences in the promoter region. Identification of the downstream regulated genes is the key to understanding the molecular mechanism of metabolic homeostasis.
Certain drought stress induced gene promoters possess a DRE/CRT motif where ABA-independent DREB/CBF transcription factors bind and act as a linking modules for ABRE in ABA-dependent gene expression [10]. It has also been reported that DREB1A/CBF3, DREB2A and DREB2C proteins interact with $A R E B / A B F$ [11]. Indicating the existence of a cross talk mechanism between ABAdependent and independent signaling events.

It is also imperative to add here that plant growth regulators play an integral role in a plant's growth and development. These growth regulators do regulate various metabolic activities so as to attune the processes involved in plant growth with the external stimuli. To this extent plant growth regulator act synergistically with transcription factors to fine tune a plants response to external stresses. Because plants need to change their growth dynamics in response to the environmental conditions, the changes in hormone balance and their underlying signaling events are the key to survival. For example, abscisic acid (ABA) and gibberellin (GA) antagonistically mediate diverse plant metabolic processes such as seed dormancy, shoot elongation, root development, flowering time etc and thus a right balance between ABA and GA is essential for plant growth and development. Although, there is unambiguous proof of the role of these plant growth regulators, but the molecular mechanisms underlying ABA-GA antagonism are far from completely understood. Studies indicate that two APETALA 2 (AP2)-domain containing transcription factors (ATFs), ABI4 in Arabidopsis and OsAP2-39 in rice, play key roles in $\mathrm{ABA}$ and $\mathrm{GA}$ antagonism [8]. In the current review, we summarize the current state of understanding of the role of AP2/EREBP transcription factors in response to environmental stresses.

\section{Structure-Function Relationship of AP2/EREBP Transcription Factors}

AP2/ EREBP family of transcription factor genes are unique to plants and the transcription factors encoded by this family are characterized by the presence of highly conserved AP2/ERF DNA binding domain $[12,13]$. The APETALA2/ethylene-responsive element binding protein (AP2/EREBP) transcription factors constitute one of the largest and most conserved gene families in plants. They are characterized by their AP2 domain comprising of 6070 amino acids and are classified into four main subfamilies: the APETALA2 (AP2), Related to ABI3/VP1 (RAV), Dehydration-Responsive Element Binding protein (DREB) and Ethylene-Responsive Factor (ERF) subfamilies [14]. AP2/ERF gene family in Arabidopsis thaliana, has been reported on the basis of number of 


\section{Cell \& Cellular Life Sciences Journal}

repetitions and domain sequence of the AP2 [13]. They distributed the Arabidopsis 144 AP2 members, into five sub-families, namely DREB, ERF, AP2, RAV and others. The DREB and ERF subfamilies, containing approximately 120 proteins, have one single AP2 domain and a conserved WLG motif. Fourteen proteins have two AP2 repetitions and belong to the AP2 subgroup. The RAV transcription regulators (six members) have a B3 DNA binding domain following the AP2 domain. The four AP2/ERF members of the other subfamily have a single AP2 repetition but lack the WLG motif characteristic of DREB and ERF proteins. While another study suggests that AP2/EREBP genes are divided into two subfamilies in Arabidopsis thaliana: AP2 genes with two AP2 domains and EREBP genes with a single AP2/ERF (Ethylene Responsive Element Binding Factor) domain [15]. However, proteins encoded by the AP2/EREBP supergene family are defined by the AP2/ERF domain, which consists of 50-60 amino acids, and these proteins are produced throughout the life cycle of a plant and are involved in a variety of regulatory mechanisms. The DNAbinding domain was first reported in the homeotic gene AP2 that regulated flower and seed development in Arabidopsis, while others have reported EREBPs in tobacco [17]. Researchers have identified three proteins (Pti-4, Pti-5 and Pti-6) interacting with the tomato disease resistance protein Pto (Pseudomonas tomato resistance gene) having a conserved domain that was similar to the domain in EREBP transcription factor (a subgroup of the AP2/ERF family) [16-18]. These observations indicate that AP2/ EREBP regulatory proteins are not only involved in the control of primary and secondary metabolism, growth and developmental programs but are also induced in response to environmental stimuli.

\section{Origin and Evolution of Genes Encoding the AP2/EREBP-TFs}

The AP2/ERF transcription factor members are distinguished by the presence of at least one copy of the AP2 sequence, which binds DNA. Interestingly, the AP2 family is highly plant specific, as sequence similarity has not been reported from animals [19]. However, lately insilico search for AP2 domain homologs from outside the plant kingdom have also been successful. In addition to identifying the three proteins from $\mathrm{T}$. thermophila, scientists detected AP2 homologs in the cyano-bacterium Trichodesmium erythraeum and in viruses' enter-bacteria phage RB49 and bacteriophage Felix 01. It is demonstrated that the cyanobacterium AP2 domain binds preferentially to DNA substrates with poly $(\mathrm{G}) /$ poly(C) stretches [20]. Presence of multiple $G$ and $C$ residues in the binding sites of plant AP2 domain suggest their functional conservedness. In the non-plant species, like ciliates, bacteria or viruses, it has been found that AP2 domain and homing endonucleases are encoded by the same genes. These identified proteins bearing an AP2 domain are predicted $\mathrm{HNH}$ endonucleases characterized by conserved His and Asn residues [21,22]. These are characterized by some important features such as they transpose from one site to another, they duplicate themselves, exploiting a homing process performed by host cell repair mechanisms, and they move extensively via lateral gene transfer [23-25]. Homing endonucleases are functionally similar to the transposable elements. Magnani, et al. [20] found that amino acid sequence similarity between one Arabidopsis AP2 domain (At4g39780 DREB protein) and the cyanobacterium T. erythraeum protein extends beyond AP2 into the $\mathrm{HNH}$ (endonuclease) domain. Therefore, the most likely and most intriguing scenario of lateral transfer of the $\mathrm{HNH}$ : AP2 gene is from bacteria or ciliates to plants. In support of this scenario, Magnani, et al. [20] noted that the vast majority of AP2/ERF proteins lack introns (122/145) as would be expected for a gene acquired, in part, from prokaryotes [20].

The evolution of AP2 domain present in various transcription factors can be explained by the presence of this domain outside plants. According to the distribution of the AP2 domains among living organisms, three possible hypotheses have been drawn: convergent evolution, divergent evolution followed by multiple loss of the character, or lateral gene transfer. To account for the widespread occurrence of the AP2 domain in plant genomes, Magnani, et al. [20] proposed that the transfer could have occurred during the evolution of chloroplasts from the endosymbiosis of an ancestral cyanobacterium [20]. Subsequent movement of cyanobacterial genes to the plant nuclear genome followed by inactivation and loss of what would probably be a harmful endonuclease activity would account for the movement of the pioneer AP2 domain from prokaryotes to an ancestor of modern plants. Alternatively, the HNH: AP2 gene might have undergone many lateral transfer events later in plant evolution [26].

\section{Role in Development}

Most cellular and developmental processes are controlled by gene expression resulting from a mobilization of multiple different sets of transcription factors (TFs) [27,28]. AP2/EREBP genes have been implicated in controlling Arabidopsis flowering and seed development, especially in the specification of organ and meristem identity [12]. Several AP2 subfamily members 


\section{Cell \& Cellular Life Sciences Journal}

(At2g28550, At3g54990 and At5g60120) are known to be the targets of miRNA172 and as part of the Arabidopsis flowering time control mechanism [29,30]. At1g12610, a DREB subgroup member confers delayed-flowering phenotype when overexpressed in Arabidopsis plants [31]. Hormonal or environmental activation patterns for several known TFs, such as At4g25490, At3g23240, At3g11020, At4g25480, and At5g05410 are also supported by previous findings in the literature for CBF1/DREB1B, ERF1, DREB2B, DERB1A/CBF3 and DREB2A, respectively $[32,33,13]$. Senescence related genes have also been reported to be controlled by an array of transcription factors including AP2/EREBP, and several of them also participate in biotic or abiotic stress responses, indicating that senescence is an integrated response of plants to endogenous developmental signals [34].

\section{Response to Environmental Stresses}

Environmental stresses constitute one of the major impediments in plant growth and productivity and are responsible for as much as fifty per cent of the yield losses in plants. AP2/EREBP family of transcription factors is involved in response to a variety of biotic (pathogen infection) as well as abiotic stresses like salinity, temperature, drought etc $[35,36,14]$. The sub-families ERF and DREB proteins play significant roles in plant responses to drought and salinity. Initially it was known that ERF and DREB respond to biotic and abiotic stresses respectively (Figure 1). TFs encoded by genes in the DREB subfamily play an important role in the resistance of plants to abiotic stresses by recognizing the dehydration- responsive element (DRE), which has a core motif of A/GCCGAC [37]. ERF subfamily genes encode a large number of ERFs, which have been shown to participate in the plant response to biotic stresses such as pathogens by recognizing the cis-acting element AGCCGCC, known as the GCC box [38]. TFs encoded by some members of this gene family bind to both GCC and the DRE cis-element [13]. ERF and DREB subfamily TFs have been identified in various plant species, including rice Arabidopsis and cotton [37]. The roles of ERF and DREB proteins in the plant response to biotic and abiotic stress have also been extensively documented [1]. AP2/EREBP transcription factor family is also responsive to cold stress with the help of CBFs (C-repeat binding factors) which can bind to cis-elements in the promoter of COR (cold responsive) genes and activate their expression [13]. Kizis, et al. have isolated two novel DRE-binding factors from maize that interact with the DRE2 element both in yeast and in maize. These proteins differ from the existing DREB1 and DREB2, as they are not homologues to them. However, it must be emphasized that not all drought stress responses are regulated by $A B A$, since a number of genes are known to be induced by various abiotic factors in ABA (ABAdeficient) and $A B I$ (ABA-insensitive) Arabidopsis mutants. Although it appears that the ABA dependent and ABA independent pathways work in isolation of each other, but it is quite possible that there is some signal networking between these two pathways. Together, these observations suggest the existence of a complex family of DRE-binding proteins with the potential to mediate distinct but interdependent responses to abiotic stresses such as drought, salt and cold.

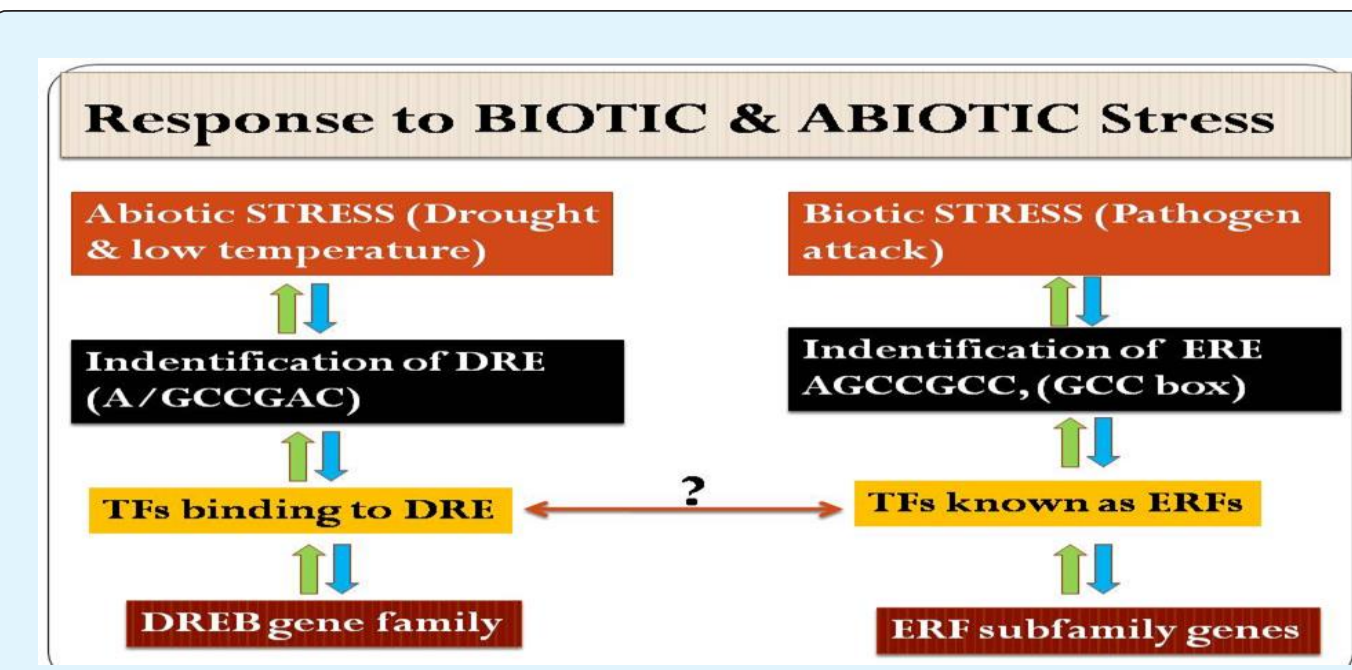

Figure 1: Response of ERF and DREB gene families to biotic and abiotic stress. 


\section{Cell \& Cellular Life Sciences Journal}

\begin{tabular}{|c|c|c|}
\hline S. No. & Sub-Family & Role \\
\hline 1 & AP2 & $\begin{array}{c}\text { Flower \& floral organ } \\
\text { development and } \\
\text { stress tolerance }\end{array}$ \\
\hline t2. & $\begin{array}{c}\text { RAV (related to } \\
\text { ABI3/VP1) }\end{array}$ & $\begin{array}{c}\text { Regulate expression } \\
\text { of target genes in } \\
\text { response to } \\
\text { environmental stress }\end{array}$ \\
\hline 3 & $\begin{array}{c}\text { ERF (ethylene } \\
\text { response factor) or } \\
\text { DREB (dehydration- } \\
\text { responsive element- } \\
\text { binding protein) }\end{array}$ & $\begin{array}{c}\text { Plant growth \& } \\
\text { development, and } \\
\text { stress tolerance } \\
\text { processes. }\end{array}$ \\
\hline
\end{tabular}

Table 1: Role of different sub-families of AP2/EREBP transcription factor.

Under biotic stress (pathogen attack) conditions, Ethylene Response Factor binds to their cis-elements (Ethylene Response Element) in the promoter region and regulates the expression of PR (Pathogenesis Responsive) genes. Researchers have performed a yeast-1-hybrid screen for identification of the drought-responsive element (DRE) as promoter cis element mediating gene expressional changes upon drought and low temperature $[37,39]$. They identified DREB1A and DREB2A as transactivating factors in the abscisic acid (ABA)-independent pathway of $A$. thaliana stress response with the core binding motif G/ACCGAC [40]. Later studies identified that several AP2/EREBP transcription factors are activated by different innervating pathways or that bind to multiple target elements [41]. For example, the DREB transcription factor TINY connects abiotic stress signalling via DRE-dependent regulation to biotic stress signalling via ERE [42].

In plants, the main families of transcription factors responsible for the regulation of genes responsive to pathogens are: i) basic leucine zipper containing domain proteins (bZIP); ii) APETALA2/ETHYLENE-RESPONSIVE ELEMENT BINDING FACTORS family (AP2/ERF); iii) WRKY family of proteins that contain either one or two 60 -amino-acid regions that contain the amino-acid sequence WRKYGQK; iv) myelocytomatosis related proteins (MYC); v) myeloblastosis related proteins (MYB) and more recently, the no apical meristem (NAM), Arabidopsis transcription activation factor (ATAF), and cup-shaped cotyledon (CUC), or also termed NAC family [7,43]. AP2/ERF transcription factors and other factors frequently act synergistically, increasing the expression of genes related to plant defence, as reported by Singh \& Buttner [44].
The role of AP2/EREBP transcription factor family has been thoroughly studied. Recent studies suggest their role in abiotic and biotic stress responses. One such study has suggested that six genes, ScDREB1, ScDREB2, ScDREB4, ScDREB6, ScDREB7, and ScDREB8 were involved in the ABA-dependent signal pathway and the desiccation, salt, and cold stress response [45]. They have reported that A5 sub-group DREB genes in S. caninervis played important roles in abiotic stresses response and enhanced stress tolerance to transgenic yeast. Transcriptome analysis revealed that APETALA2/ethylene response factor (AP2/ERF) transcription factor superfamily is responsible for cold stress in Brassica napus [46]. Another study which aimed to analyse the mechanism underlying $S$. lycopersicum ERF (SIERF) TFs in response to TYLCV infection identified a total of 22 tomato AP2/ERF TFs in response to TYLCV according to transcriptome database [47]. Additionally, transgenic rice overexpressing an AP2/ERF Type Transcription Factor OsEREBP1 confers biotic and abiotic stress tolerance in Rice. Similar studies using Medicago truncatula genome information have been conducted by Shu, et al. [48]. They identified and characterized 123 putative AP2/ERF genes, which were named as MtERF1-123. Using transcriptome, highthroughput sequencing data, and qRT-PCR analysis, the expression patterns of MtERF genes in tissues during development and under abiotic stresses has been assessed. In total, $87 \mathrm{MtERF}$ genes were expressed in plant tissues, most of which were expressed in specific tissues during development or under specific abiotic stress treatments. These results clearly suggest that MtERF genes are involved in developmental regulation and environmental responses. Furthermore, cold and freezing stress induced a cluster of DREB subfamily members on chromosome 6 suggesting that these genes might contribute to freezing tolerance to $M$. truncatula. These studies provide valuable information about the role of AP2/EREBP family of transcription factor family in abiotic and biotic stress. Further studies can be done by characterizing these genes and utilizing them to improve stress tolerance in plants.

\section{Conclusion}

The complete sequencing of several plant genomes has fuelled the ongoing research in plant molecular genetics and functional genomics. There are many new and powerful genomic tools to power the research in the field of plant genomics and proteomics. It is crucial that important information is gathered about the plant cellular, biochemical, and molecular processes that drive the responses to the change in the environment of plants. 


\section{Cell \& Cellular Life Sciences Journal}

Plants respond to various environmental stresses in a very efficient way. There are various regulatory processes in plant system that helps it to withstand the environmental changes such as hormones and membrane channels and many signalling pathways. Within this complex system, there are another group of regulatory proteins, the transcription factors, of which the AP2/EREBP family of transcription factors is just a part. The transcription factors encoded by this family find crucial role in plant's defence responses against both abiotic and biotic stresses. Over the years, yeast twohybrid assays and microarray techniques have lead to the identification of protein-protein interactions and proteinDNA interactions. This has indeed outspread our knowledge towards the network of these transcription factors and their role in regulation of stress related genes. These studies may lead to a better understanding, not only of the interactions that regulate these transcription factors but also of the important biological processes that these factors modulate.

Although much literature has been published indicating their role in regulation of genes and about their role in the defence responses in plants yet there still remains a thorough understanding of the complex signalling network that exists in plants to combat environmental stresses.

\section{Acknowledgement}

The author (AP) is thankful to the Council of Scientific and Industrial Research, Government of India for financial assistance and SA is thankful to Department of Biotechnology, Govt. of India for program mode support for R\&D in agricultural biotechnology.

\section{References}

1. Agarwal M, Hao Y, Kapoor A, Dong $\mathrm{CH}$, Fujii $\mathrm{H}$, et al. (2006) A R2R3 type MYB transcription factor is involved in the cold regulation of CBF genes and in acquired freezing tolerance. J Biol Chem 281(49): 37636-37645.

2. Cushman JC, Bohnert HJ (2000) Genomic approaches to plant stress tolerance. Curr Opin Plant Biol 3(2): 117-124.

3. Gao G, Zhong Y, Guo A, Zhu Q, Tang W, et al. (2006) DRTF: a database of rice transcription factors. Bioinformatics 22(10): 1286-1287.
4. Todaka D, Nakashima K, Shinozaki K, Shinozaki KY (2012) Towards understanding transcriptional regulatory networks in abiotic stress responses and tolerance in rice. Rice 5(1): 6.

5. Khong GN, Richaud F, Coudert Y, Pati PK, Santi C, et al. (2008) Modulating rice stress tolerance by transcription factors. Biotechnol Genet Eng Rev 25: 381-403.

6. Zhu JK (2002) Salt and drought stress signal transduction in plants. Annu Rev Plant Biol 53: 247273.

7. Singh K, Foley RC, Oñate-Sánchez L (2002) Transcription factors in plant defense and stress responses. Curr Opin Plant Biol 5(5): 430-436.

8. Joshi R, Wani SH, Singh B, Bohra A, Dar ZA, et al. (2016) Transcription factors and plants response to drought stress: current understanding and future directions. Front plant sci 7: 1029.

9. Nakashima K, Jan A, Todaka D, Maruyama K, Goto S, et al. (2014) Comparative functional analysis of six drought-responsive promoters in transgenic rice. Planta 239(1): 47-60.

10. Singh D, Laxmi A (2015) Transcriptional regulation of drought response: a tortuous network of transcriptional factors. Front. Plant Sci 6: 895.

11. Lee SJ, Kang JY, Park HJ, Kim MD, Bae MS, et al. (2010) DREB2C interacts with ABF2, abZIP protein regulating abscisic acid-responsive gene expression, and it's over expression affects abscisic acid sensitivity. Plant Physiol 153(2): 716-727.

12. Riechmann JL, Meyerowitz EM (1998) The AP2/EREBP family of plant transcription factors. Biol Chem 379(6): 633-646.

13. Sakuma Y, Liu Q, Dubouzet JG, Abe H, Shinozaki K, et al. (2002) DNA-binding specificity of the AP2/ERF domain of Arabidopsis DREBs, transcription factors involved in dehydration-and cold-inducible gene expression. Biochem Biophys Res Commun 290(3): 998-1009.

14. Liu C, Zhang T (2017) Expansion and stress responses of the AP2/EREBP superfamily in cotton. BMC Genomics. 18(1): 118. 


\section{Cell \& Cellular Life Sciences Journal}

15. Shigyo M, Hasebe $M$, Ito $M$ (2006) Molecular evolution of the AP2 subfamily. Gene 366(2): 256-65.

16. Jofuku KD, den Boer BGW, Van Montagu M, Okamuro JK (1994) Control of Arabidopsis flower and seed development by the homeotic gene APETALA2. Plant Cell 6(9): 1211-1225.

17. Ohme-Takagi M, Shinshi H (1995) Ethylene-inducible DNA binding proteins that interact with an ethyleneresponsive element. Plant Cell 7(2): 173-182.

18. Zhou J, Tang X, Martin GB (1997) The Pto kinase conferring resistance to tomato bacterial speck disease interacts with proteins that bind a ciselement of pathogenesis-related genes. EMBO J 16(11): 3207-3218.

19. Krizek BA (2003) AINTEGUMENTA utilizes a mode of DNA recognition distinct from that used by proteins containing a single AP2 domain. Nucleic Acids Res 31(7): 1859-1868.

20. Magnani E, Sjölander K, Hake S (2004) From endonucleases to transcription factors: evolution of the AP2 DNA binding domain in plants. Plant Cell 16: 2265-2277.

21. Shub DA, Goodrich-Blair H, Eddy SR (1994) Amino acid sequence motif of group I intron endonucleases is conserved in open reading frames of group II introns. Trends Biochem Sci 19: 402-404

22. Dalgaard JZ, Klar AJ, Moser MJ, Holley WR, Chatterjee A, et al. (1997) Statistical modeling and analysis of the LAGLIDADG family of site-specific endonucleases and identification of an intein that encodes a site-specific endonuclease of the HNH family. Nucleic Acids Res 25(22): 4626-4638.

23. Gimble FS (2000) Invasion of a multitude of genetic niches by mobile endonuclease genes. FEMS Microbiol Lett 185: 99-107.

24. Chevalier BS, Stoddard BL (2001) Homing endonucleases: Structural and functional insight into the catalysts of intron/intein mobility. Nucleic Acids Res 29(18): 3757-3774.

25. Koufopanou V, Goddard MR, Burt A (2002) Adaptation for horizontal transfer in a homing endonuclease. Mol Biol Evol 19(3): 239-246.
26. Wessler SR (2005) Homing into the origin of the AP2 DNA binding domain. Trends plant sci 10(2): 54-56.

27. Grandori C, Cowley SM, James LP, Eisenman RN (2000) The Myc/Max/Mad network and the transcriptional control of cell behavior. Annu Rev Cell Dev Biol 16: 653-699.

28. Stracke R, Werber M, Weisshaar B (2001) The R2R3MYB gene family in Arabidopsis thaliana. Curr Opin Plant Biol 4(5): 447-456.

29. Aukerman MJ, Sakai H (2003) Regulation of flowering time and floral organ identity by a microRNA and its APET ALA2-like target genes. Plant Cell 15(11): 27302741.

30. Schmid M, Uhlenhaut NH, Godard F, Demar M, Bressan R, et al. (2003) Dissection of floral induction pathways using global expression analysis. Development 130(24): 6001-6012.

31. Magome H, Yamaguchi S, Hanada A, Kamiya Y, Oda K (2004) Dwarf and delayed-flowering 1, a novel Arabidopsis mutant deficient in gibberellin biosynthesis because of overexpression of a putative AP2 transcription factor. Plant J 37(5): 720-729.

32. Stockinger EJ, Gilmour SJ, Thomashow MF (1997) Arabidopsis thaliana CBF1 encodes an AP2 domaincontaining transcriptional activator that binds to the C-repeat/ DRE, a cis-acting DNA regulatory element that stimulates transcription in response to low temperature and water deficit. Proc Natl Acad Sci USA 94(3): 1035-1040.

33. Solano R, Stepanova A, Chao Q, Ecker JR (1998) Nuclear events in ethylene signaling: a transcriptional cascade mediated by ETHYLENE-INSENSITIVE3 and ETHYLENE- RESPONSE-FACTOR1. Genes Deve 12(23): 3703-3714.

34. Safavi-Rizi V, Franzaring J, Fangmeier A, Kunze R (2018) Divergent N Deficiency-Dependent Senescence and Transcriptome Response in Developmentally Old and Young Brassica napus Leaves. Front Plant Sci 9: 48.

35. Dietz KJ, Vogel MO, Viehhauser A (2010) AP2/EREBP transcription factors are part of gene regulatory networks and integrate metabolic, hormonal and environmental signals in stress acclimation and retrograde signalling. Protoplasma 245(1-4): 3-14. 


\section{Cell \& Cellular Life Sciences Journal}

36. Balan B, Marra FP, Caruso T, Martinelli F (2018) Transcriptomic responses to biotic stresses in Malus x domestica: a meta-analysis study. Sci Rep 8: 1970.

37. Liu Q, Kasuga M, Sakuma Y, Abe H, Miura S, et al. (1998) Two transcription factors, DREB1 and DREB2, with an EREBP/AP2 DNA binding domain separate two cellular signal transduction pathways in droughtand low temperature-responsive gene expression, respectively, in Arabidopsis. Plant Cell 10(8): 13911406.

38. Fujimoto SY, Ohta M, Usui A, Shinshi H, Ohme-Takagi M (2000) Arabidopsis ethylene-responsive element binding factors act as transcriptional activators and repressors of GCC box-mediated gene expression. Plant Cell 12(3): 393-404.

39. Guo HW, Ecker JR (2004) The ethylene signaling pathway: new insights. Curr Opin Plant Biol 7(1): 4049.

40. Shinozaki K, Yamaguchi-Shinozaki K (2000) Molecular responses to dehydration and low temperature: differences and cross-talk between two stress signaling pathways. Curr Opin Plant Biol 3(3): 217-223.

41. Lorenzo O, Piqueras R, Sanchez-Serrano JJ, Solano R (2003) ETHYLENE RESPONSE FACTOR1 integrates signals from ethylene and jasmonate pathways in plant defense. Plant Cell 15(1): 165-178.

42. Sun S, Yu JP, Chen F, Zhao TJ, Fang XH, et al. (2008) TINY, a dehydration-responsive element (DRE)- binding protein like transcription factor connecting the DRE- and ethylene responsive element-mediated signaling pathways in Arabidopsis. J Biol Chem 283(10): 6261-6271.

43. Van Verk MC, Gatz C, Linthorst HJM (2009) Transcriptional regulation of plant defense responses. Adv Bot Res 51: 397-438.

44. Singh KB, Buttner M (1997) Arabidopsis thaliana ethylene-responsive element binding protein (AtEBP), an ethylene-inducible, GCC box DNA-binding protein interacts with an ocs element binding protein. Proc Natl Acad Sci USA 94(11): 5961-5966.

45. Li H, Zhang D, Li X, Guan K, Yang H (2016) Novel DREB A-5 subgroup transcription factors from desert moss (Syntrichia caninervis) confers multiple abiotic stress tolerance to yeast. J plant physiol 194: 45-53.

46. Du C, Hu K, Xian S, Liu C, Fan J, et al. (2016) Dynamic transcriptome analysis reveals AP2/ERF transcription factors responsible for cold stress in rapeseed (Brassica napus L.). Mol Genet Genomics 291(3): 1053-1067.

47. Huang Y, Zhang BL, Sun S, Xing GM, Wang F, et al. (2016) AP2/ERF Transcription Factors Involved in Response to Tomato Yellow Leaf Curly Virus in Tomato. Plant Genome 9(2).

48. Shu Y, Liu Y, Zhang J, Song L, Guo C (2015) Genomewide analysis of the AP2/ERF superfamily genes and their responses to abiotic stress in Medicago truncatula. Front Plant Sci 6: 1247. 